\title{
Awareness of Olfactory Dysfunction in Subjective Cognitive Decline, Mild Cognitive Decline, and Alzheimer's Disease
}

\author{
R. Tahmasebi ${ }^{1} \cdot$ S. Zehetmayer ${ }^{2}$ E. Stögmann ${ }^{1} \cdot$ Johann Lehrner ${ }^{1}$ \\ Received: 7 January 2019 / Accepted: 5 June 2019 / Published online: 24 June 2019 \\ (C) The Author(s) 2019
}

\begin{abstract}
Introduction Hyposmia and metacognitive errors are related to aging, depression, male gender, and cognitive decline. The current study investigated the awareness of olfactory dysfunction in subjective cognitive decline (SCD), mild cognitive impairment (MCI), and Alzheimer's disease (AD), as well as the influence of additional factors.

Methods A sample of 641 patients, including controls, SCD, non-amnestic MCI (naMCI), amnestic MCI (aMCI), and AD patients, was assessed with the Sniffin' Sticks odor identification test (OIT) and the subjective olfactory capability (SOC) scale, in addition to measures of depressive symptoms, verbal memory, and executive functioning. Olfactory awareness groups were formed by means of the cutoffs of the OIT and the SOC.

Results Moderate and small, although significant, correlations between the OIT and the SOC were found among the study groups, with a significant discrimination of measured olfactory function via subjective assessment existing among controls but not among patients with $\mathrm{AD}$. Of all AD patients, $34 \%$ overrated their sense of smell while $21 \%$ correctly identified themselves as being hyposmic, as opposed to corresponding $6 \%$ and $1 \%$ of healthy elderly. Overraters and correct hyposmic participants showed higher age and worse verbal memory and executive functions.

Conclusions Reduced odor identification might underlie the same pathological changes within the brain as cognitive impairment and could serve as an additional marker for the development of AD.

Implications Although people with $\mathrm{AD}$ are aware of hyposmia to some extent, the majority is affected by overestimation of the ability to smell, making the combination of subjective ratings and measures of olfactory function an interesting topic for further research.
\end{abstract}

Keywords Cognitive dysfunction $\cdot$ Neuropsychology $\cdot$ Olfaction $\cdot$ Memory

\section{Introduction}

Several studies carried out during the last few years have been trying to find a reliable way of predicting the development of Alzheimer's disease (AD) among patients with mild cognitive impairment (MCI). MCI can be described as an intermediate state between age-related cognitive deterioration and dementia and is referred to as amnestic MCI (aMCI) if the impairment affects tasks requiring a normal function of memory or as nonamnestic MCI (naMCI) if other cognitive areas are impaired (Petersen 2004). Recently, these studies started focusing on

Johann Lehrner

johann.lehrner@meduniwien.ac.at

1 Department of Neurology, Medical University of Vienna, Währinger Gürtel 18-20, A-1097 Wien, Austria

2 Center for Medical Statistics, Informatics, and Intelligent Systems, Medical University of Vienna, Vienna, Austria olfactory dysfunction as a potential marker for the development of $\mathrm{AD}$ in patients suffering from MCI (Lehrner et al. 1997; Murphy 2002; Djordjevic et al. 2008; Devanand et al. 2015). Additionally, a new diagnostic entity, called subjective cognitive decline (SCD), has been gaining more attention lately as a potential prodromal stage of MCI and AD (Jessen et al. 2014).

In general, olfactory deficits are positively related to increasing age (Doty and Kamath 2014), to male sex (Kern et al. 2014), and to cognitive decline, especially concerning memory deficits (Lehrner et al. 2009; Wehling et al. 2016). Interestingly, the majority of the concerned population is not aware of any impairment of their ability to perceive odors (Nordin et al. 1995; Murphy et al. 2002; Bahar-Fuchs et al. 2011). Moderate correlations between measures and subjective ratings of the ability to smell are often explained by the partly subconscious nature of olfactory perception compared with other human senses. The more gradually hyposmia emerges, such as through aging or neurodegenerative 
diseases, the more likely people overrate their olfactory capacity (White and Kurtz 2003). Overestimation as well as underestimation of abilities is the result of deficits in metacognitive knowledge, which is called anosognosia (Rosen 2011), a phenomenon relatively common in neurodegenerative diseases (Lehrner et al. 2015; White et al. 2016). Specifically, olfactory awareness is negatively related to age (Wehling et al. 2011), cognitive decline, especially concerning memory and executive control such as attention (Devanand et al. 2000; Wehling et al. 2011), and male sex (Wehling et al. 2011).

The main purpose of this study is to investigate the awareness of olfactory dysfunction in people with SCD, MCI, and $\mathrm{AD}$ as well as factors influencing it. First, severity and prevalence rates of olfactory impairment by means of a smell performance test as well as a subjective rating will be compared in healthy controls and subjects with SCD, naMCI, aMCI, and AD. As shown in previous studies, it can be expected that patients with cognitive impairment score lower on the measure of olfactory function than controls (Djordjevic et al. 2008; Lehrner et al. 2009; Bahar-Fuchs et al. 2011; Woodward et al. 2017), but not on the subjective one (Nordin et al. 1995; Lehrner et al. 2009; Bahar-Fuchs et al. 2011).

Second, the question of validity of self-reported olfactory functioning as an economic means of screening in neurodiagnostics within controls and $\mathrm{AD}$ patients and the relation between measured scores and subjective rating of olfactory ability, representing olfactory awareness, within the diagnostic groups, will be explored. So far, previous studies on the self-rated ability to smell led to conflicting results regarding these purposes (Devanand et al. 2000; Bahar-Fuchs et al. 2011).

Third, variables of cognitive decline, namely verbal memory and executive control, in addition to depressive symptoms, age, and gender, will be compared between formed groups of awareness of olfactory functioning in the study population. The awareness of one's own olfactory functioning is likely to be not only influenced by symptoms of depression and age, but also by cognitive decline, in a way that depressed patients tend to underestimate their abilities (Hur et al. 2018; Rochet et al. 2018), while the contrary is expected with increasing age and cognitive decline (Nordin et al. 1995; Devanand et al. 2000; Wehling et al. 2011).

\section{Method}

\section{Participants}

The study was conducted in accordance with the Helsinki Declaration and approved by the Ethical Committee of the Medical University of Vienna. The study site was the Department of Neurology of the Medical University of
Vienna. Patients were either referred by general practitioners, neurologists, or psychiatrists or were self-referrals. All patients received a complete neurological examination, standard laboratory blood tests, and psychometric testing (Pusswald et al. 2013), which included the following tests: the MiniMental State Examination (MMSE) (Folstein et al. 1975); the Wortschatztest (WST), a vocabulary IQ test (Schmidt and Metzler 1992); the Beck Depression Inventory II (BDIII) (Hautzinger et al. 2006); and the Neuropsychological Test Battery Vienna (NTBV), which includes assessments of psychomotor speed, attention, language, memory, and executive functions (Lehrner et al. 2007). In most cases, a computer tomography scan or magnetic resonance imaging scan of the brain was obtained (Lehrner et al. 2015).

The study population consisted of 641 persons, among them 161 cognitively healthy controls, 69 SCD, 210 naMCI, $163 \mathrm{aMCI}$, and $38 \mathrm{AD}$ patients. The diagnosis of SCD, naMCI, aMCI, or AD was determined by the decision of a consensus committee including neuropsychologists, neurologists, and other study personnel involved in the evaluation of the patients' cognitive status. Diagnosis of MCI was based on Petersen criteria (Petersen 2004) and required a $z$ score of 1.5 SD below the age and education-corrected norms for elderly controls using MIN-mode MCI classification (Pusswald et al. 2013; Lehrner et al. 2015). SCD classification required (a) the presence of subjective memory deterioration as manifested by the seeking of medical help for memory problems and (b) the concurrent absence of any objectively measurable cognitive deficits (mean $z$ score of each domain greater than 1.5 SD) (Jessen et al. 2014; Lehrner et al. 2015). AD was diagnosed according to the National Institute of Neurological and Communicative Disorders and Stroke and the Alzheimer's Disease and Related Disorders Association (NINCDS-ADRDA) (McKhann et al. 1984) and DSM-IV criteria ( $\mathrm{Sa} ß$ et al. 1996). None of the olfactory assessments evaluated by this study were used for diagnostic purposes regarding the study population.

Cognitively healthy control subjects were recruited via advertisements. They underwent a rigorous screening evaluation and were assessed as being in good health. The criteria for healthy function included (a) an MMSE score $\geq 27$ and a Montreal Cognitive Assessment score $\geq 26$, adjusted for education; (b) no active neurological or psychiatric disease; (c) no psychotropic medication; and (d) no medical disorder or treatment that negatively affects cognitive functioning. Controls underwent the same neuropsychological assessment as patients (Lehrner et al. 2015).

The exclusion criteria for all participants were as follows: (1) evidence of stroke as determined by neuroradiologic and clinical examination; (2) history of severe head injury; (3) current psychiatric diagnosis according to ICD-10, however, patients with (sub-) depressive symptoms were included because (sub-) depressive symptoms often occur in elderly 
patients; and (4) any medical condition that leads to severe cognitive deterioration including renal, respiratory, cardiac, and hepatic disease (Lehrner et al. 2015). Exclusion criteria were applied during routine medical checkup. Thus, no exact data regarding the number of excluded patients is available.

\section{Measures}

Olfactory functioning was measured as well as self-rated by the patients and the combination of both served as an indication for the associated awareness.

To measure olfactory performance, the odor identification test (OIT) of the Sniffin' Sticks test battery (Kobal et al. 1996) was administered. It consists of 16 pen-like sticks containing different odors that are each to be identified within a four-alternative forced-choice format. The Sniffin' Sticks test kit has established normative data (Hummel et al. 2007) and is frequently used in age-related clinical assessments (Hummel et al. 1997). Scores range from 0 to 16 depending on how many odors were correctly identified, and according to Hummel et al., a score of 9 and below is considered as being hyposmic/anosmic for patients aged from 51 to 70 years, which equals the average age of our study population (Hummel et al. 2007).

To capture the subjective rating of one's own sense of smell, the subjective olfactory capability (SOC) domain of the assessment of self-reported olfactory functioning and olfaction-related quality of life (ASOF) (Pusswald et al. 2012) was administered. It consists of one question, namely "How would you rate your sense of smell over the past four weeks?," which is answered by circling a number on a scale from 0 (unable to smell) to 10 (best possible). A score of 3 and below is considered as subjective impairment in olfactory functioning. The ASOF has proven to be of good validity and reliability in normosmic controls as well as in hyposmic/anosmic patients (Pusswald et al. 2012).

The NTBV (Lehrner et al. 2007) measures different aspects of cognitive decline and was administered to extensively evaluate the domains of attention, executive functions, language, and memory. Part of the NTBV are the Verbal Selective Reminding Test (VSRT) (Lehrner et al. 2006) and the Trail Making Test (TMT) with part A and B (Reitan 1979), measuring verbal memory and executive functions, respectively.

Finally, depressive symptoms were detected by the BDI-II (Hautzinger et al. 2006). The BDI-II is a questionnaire broadly applied in clinical research because of its high internal consistency, test-retest reliability, and validity (Wang and Gorenstein 2013). Scores range from 0 to 63 with a score above 13 indicating clinical depression.

\section{Data Preparation and Statistical Methods}

All statistics have been calculated with IBM SPSS Statistics Version 23. The significance level for all tests, unless stated otherwise, was set to $\alpha=0.05$. According to visual inspection of histograms and the Kolmogorov-Smirnov test, none of the variables ascertained for this study showed a normal distribution, which is why non-parametric tests were used for their evaluation.

Concerning the first hypothesis, the subjective rating and the measure of olfactory function were compared across diagnostic groups via a Kruskal-Wallis test and pairwise post hoc testing using Dunn's test was applied for variables with significant differences. Prevalence rates of hyposmia/anosmia were calculated by means of the abovementioned cutoffs of the OIT and the SOC domain, respectively, which were then used to split the awareness of olfactory function into the following four categories: (1) underestimation of olfactory function: normosmia according to OIT and hyposmia according to SOC, (2) awareness of normosmia: normosmia according to OIT and normosmia according to SOC, (3) awareness of hyposmia: hyposmia according to OIT and hyposmia according to SOC, and (4) overestimation of olfactory function: hyposmia according to OIT and normosmia according to SOC. Due to the low number of patients in the individual combinations of cognitive and olfactory function groups, the use of inferential statistics was not feasible for the evaluation of this hypothesis.

To examine the relationship between measured and selfrated olfactory performance, Spearman rank correlations of the OIT and the SOC were performed and compared between the diagnostic groups. Furthermore, to investigate the sensitivity and specificity of the SOC domain, receiver operating characteristic (ROC) curves were created for AD patients as well as for healthy subjects, using the odor identification score as a gold standard. The areas under the curves were explored and compared for conclusions concerning the validity of the SOC domain.

To test for differences in age, gender, BDI-II, VSRT delayed recall, and TMT B-A score between the awareness groups, a Kruskal-Wallis and Pearson's chi-squared test were conducted. For all variables with a significant difference during the aforementioned tests, pairwise post hoc testing was conducted via Dunn's test or via Fisher's exact test with Bonferroni correction.

\section{Results}

\section{Sample Characteristics of the Study Population}

Table 1 shows the sample characteristics of the study population and their comparison among all diagnostic 
Table 1 Sample characteristics

\begin{tabular}{|c|c|c|c|c|c|c|}
\hline & \multicolumn{5}{|c|}{ Median (25th percentile/75th percentile) } & \multirow{2}{*}{$\begin{array}{l}p \text { value of } \\
\text { Kruskal-Wallis } \\
\text { test }\end{array}$} \\
\hline & $\begin{array}{l}\text { Control subjects } \\
(n=161)\end{array}$ & $\begin{array}{l}\text { Patients with SCD } \\
(n=69)\end{array}$ & $\begin{array}{l}\text { Patients with naMCI } \\
(n=210)\end{array}$ & $\begin{array}{l}\text { Patients with aMCI } \\
(n=163)\end{array}$ & $\begin{array}{l}\text { Patients with AD } \\
(n=38)\end{array}$ & \\
\hline Age & $65(57 / 73)^{\mathrm{c}, \mathrm{d}, \mathrm{e}}$ & $66(58.5 / 74)^{\mathrm{e}}$ & $69(61 / 74)^{\mathrm{a}, \mathrm{e}}$ & $69(61 / 75)^{\mathrm{a}, \mathrm{e}}$ & $74.5(67 / 77)^{\mathrm{a}, \mathrm{b}, \mathrm{c}, \mathrm{d}}$ & 0.001 \\
\hline Sex & $62.7 \%$ Female & $50.7 \%$ Female & $64.3 \%$ Females & $53.4 \%$ Female & $55.3 \%$ Female & $\mathrm{NA}^{*}$ \\
\hline Education & $11(9 / 15)$ & $12(9 / 16)$ & $11(8 / 16)$ & $12(8 / 17)$ & $9(8 / 12)$ & 0.063 \\
\hline MMSE & $29(28 / 30)^{\mathrm{c}, \mathrm{d}, \mathrm{e}}$ & $29(28 / 30)^{\mathrm{c}, \mathrm{d}, \mathrm{e}}$ & $28(27 / 29)^{\mathrm{a}, \mathrm{b}, \mathrm{d}, \mathrm{e}}$ & $28(27 / 29)^{\mathrm{a}, \mathrm{b}, \mathrm{c}, \mathrm{e}}$ & $25(24 / 26)^{\mathrm{a}, \mathrm{b}, \mathrm{c}, \mathrm{d}}$ & $<0.001$ \\
\hline WST-IQ & $107(99 / 118)^{\mathrm{b}, \mathrm{e}}$ & $118(104.5 / 122)^{\mathrm{a}, \mathrm{d}, \mathrm{e}}$ & $110(100 / 122)^{\mathrm{e}}$ & $107(99 / 118)^{\mathrm{b}, \mathrm{e}}$ & $101(91 / 114)^{\mathrm{a}, \mathrm{b}, \mathrm{c}, \mathrm{d}}$ & 0.001 \\
\hline BDI-II & $4(2 / 8)^{b, c, d, e}$ & $7(4.5 / 13)^{\mathrm{a}}$ & $9(5 / 14)^{\mathrm{a}}$ & $9(4 / 15)^{\mathrm{a}}$ & $8(5 / 14)^{\mathrm{a}}$ & $<0.001$ \\
\hline
\end{tabular}

$S C D$ subjective cognitive decline, naMCI non-amnestic mild cognitive impairment, $a M C I$ amnestic mild cognitive impairment, $A D$ Alzheimer's disease, MMSE Mini-Mental State Examination, WST IQ Wortschatztest IQ, BDI-II Beck Depression Inventory II

${ }^{a}$ Significantly different $(\alpha=0.05)$ from controls according to Mann-Whitney $U$ test

${ }^{\mathrm{b}}$ Significantly different $(\alpha=0.05)$ from SCD according to Mann-Whitney $U$ test

${ }^{\mathrm{c}}$ Significantly different $(\alpha=0.05)$ from naMCI according to Mann-Whitney $U$ test

${ }^{\mathrm{d}}$ Significantly different $(\alpha=0.05)$ from aMCI according to Mann-Whitney $U$ test

${ }^{\text {e }}$ Significantly different $(\alpha=0.05)$ from AD according to Mann-Whitney $U$ test

$* p=0.105$ for two-sided chi-squared test between all groups

groups via Kruskal-Wallis and Pearson's chi-squared test. For all variables with a significant difference during the aforementioned tests, pairwise testing was conducted via Mann-Whitney $U$ test or via Fisher's exact test. Age was significantly higher among AD patients compared with the other groups and significantly lower among controls compared with naMCI and aMCI patients. The MMSE and WST IQ scores of the AD group were significantly lower compared with the other groups. In general, the MMSE score differed significantly between all groups except for between controls and SCD patients, showing a decline from controls to AD patients. Interestingly, the WST IQ of the aMCI group differed significantly only from the SCD and AD group, but not from controls or naMCI patients. The SCD group had a significantly higher WST IQ than the other groups, with the exception of the naMCI group. A potential explanation for this would be that our study recruited SCD patients, as opposed to controls, from a medical help seeking rather than a population-based sample, which might have biased the selection of patients in the direction of overreporting of cognitive impairment. As the patients of our study population originate from a memory clinic, the obtained results might not be representative of the general population. Controls had a significantly lower BDI-II score compared with the other groups. There was no significant difference in the distribution of sex or the duration of education between the groups.

\section{Olfactory Identification, Self-Estimation of Olfaction, and Awareness of Olfactory Functioning}

The comparison of measured and self-rated olfactory function among diagnostic groups, which is shown in Table 2, revealed significant differences for both variables. The subsequently conducted post hoc testing revealed the following: The OIT score was significantly lower among AD patients compared with the other groups; significantly lower among aMCI patients compared with healthy controls, SCD, and naMCI patients; and significantly lower among naMCI patients compared with healthy controls. Subjective olfaction as measured by the SOC score showed a significantly lower score among $\mathrm{AD}$ patients compared with controls.

The resulting occurrences of tested and self-reported olfactory impairment as well as the prevalence of awareness groups among the diagnostic groups are displayed in Table 3. Based on the olfactory cutoff score for the OIT, the diagnostic groups of the study population displayed a steadily increasing percentage of patients with measured hyposmia when looking at them in the following order: controls $(7.5 \%) \geq \mathrm{SCD}$ $(11.6 \%) \geq \operatorname{naMCI}(19.5 \%) \geq \operatorname{aMCI}(34.4 \%) \geq \mathrm{AD}(55.3 \%)$. Total prevalence of measured hyposmia was $21.5 \%$. Using the cutoff score of the SOC, a similar order can be found, with the only difference being that the SOC test showed a lower overall prevalence of hyposmia among the study population and almost equal percentages for $\mathrm{SCD}$, naMCI, and aMCI patients. A graphical illustration of these results can be found in Fig. 1a and $b$. Regarding the prevalence of overestimation of 
Table 2 Comparison of measured and self-rated olfactory function among diagnostic groups

\begin{tabular}{|c|c|c|c|c|c|c|}
\hline & \multicolumn{5}{|c|}{ Median (25th percentile/75th percentile) } & \multirow{2}{*}{$\begin{array}{l}p \text { value of } \\
\text { Kruskal-Wallis } \\
\text { test }\end{array}$} \\
\hline & $\begin{array}{l}\text { Control subjects } \\
(n=161)\end{array}$ & $\begin{array}{l}\text { Patients with SCD } \\
(n=69)\end{array}$ & $\begin{array}{l}\text { Patients with naMCI } \\
(n=210)\end{array}$ & $\begin{array}{l}\text { Patients with aMCI } \\
(n=163)\end{array}$ & $\begin{array}{l}\text { Patients with AD } \\
(n=38)\end{array}$ & \\
\hline OIT & $13(12 / 14)^{\mathrm{c}, \mathrm{d}, \mathrm{e}}$ & $13(11 / 14)^{\mathrm{d}, \mathrm{e}}$ & $13(10.75 / 14)^{\mathrm{a}, \mathrm{d}, \mathrm{e}}$ & $11(8 / 13)^{\mathrm{a}, \mathrm{b}, \mathrm{c}, \mathrm{e}}$ & $9(7 / 11)^{\mathrm{a}, \mathrm{b}, \mathrm{c}, \mathrm{d}}$ & $<0.001$ \\
\hline SOC & $8(6 / 10)^{\mathrm{e}}$ & $8(5 / 10)$ & $8(6 / 10)$ & $7(5 / 9.5)$ & $6(2.75 / 9)^{\mathrm{a}}$ & 0.007 \\
\hline
\end{tabular}

Ranges in the study population: OIT $0-16$, SOC $0-10$

$S C D$ subjective cognitive decline, naMCI non-amnestic mild cognitive impairment, $a M C I$ amnestic mild cognitive impairment, $A D$ Alzheimer's disease, $O I T$ odor identification test, $S O C$ subjective olfactory capability

${ }^{\text {a }}$ Significantly different from controls according to Dunn's test

${ }^{\mathrm{b}}$ Significantly different from SCD according to Dunn's test

${ }^{\mathrm{c}}$ Significantly different from naMCI according to Dunn's test

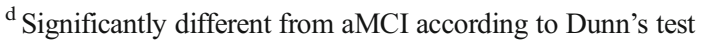

${ }^{\text {e }}$ Significantly different from AD according to Dunn's test

olfactory function and of the awareness of hyposmia among patient groups, a similar sequence can be found. The frequency of the awareness of normosmia showed an opposite order, decreasing from controls to $\mathrm{AD}$ patients. The frequency of the underestimation of olfactory function did not show such a strict order, with the aMCI group having the lowest and the $\mathrm{AD}$ group having the highest percentage of patients with underestimation. Regarding the entire patient sample, awareness of normosmia was the most prevalent, followed by overestimation of olfaction, awareness of hyposmia, and underestimation of olfactory capability. For a further, purely descriptive illustration of the awareness of olfactory dysfunction among diagnostic groups, $z$ scores of the OIT and SOC scores have been calculated for every patient based on the mean and standard deviation values of the entire sample. The mean difference between these two $z$ scores (awareness of olfactory dysfunction $=z$ score SOC $-z$ score OIT) can be seen in Fig. 2 for each diagnostic group. Patients with aMCI and AD showed a slightly positive mean value, indicating overestimation of olfactory function, while controls showed a slightly negative mean value, which indicates underestimaion of olfactory function. SCD and even more so naMCI patients showed values close to zero, indicating correct self-estimation of olfactory function. The $\mathrm{AD}$ group showed the highest standard error of the mean awareness score, probably due to the lower number of patients compared with the other groups.

\section{Validation of the Subjective Olfactory Capability Domain}

To investigate the sensitivity and specificity of the SOC domain in relation to achievements on the OIT, ROC curves were created with the dichotomized OIT score (hyposmic/anosmic versus normosmic) as a state variable. This was done

Table 3 Prevalences of olfactory impairment and olfactory awareness groups among diagnostic groups

\begin{tabular}{lllllll}
\hline & $\begin{array}{l}\text { Control subjects } \\
(n=161)\end{array}$ & $\begin{array}{l}\text { Patients with SCD } \\
(n=69)\end{array}$ & $\begin{array}{l}\text { Patients with naMCI } \\
(n=210)\end{array}$ & $\begin{array}{l}\text { Patients with aMCI } \\
(n=163)\end{array}$ & $\begin{array}{l}\text { Patients with AD } \\
(n=38)\end{array}$ & $\begin{array}{l}\text { Total sample } \\
(n=641)\end{array}$ \\
\hline $\begin{array}{c}\text { Measured } \\
\text { hyposmia }\end{array}$ & $12(7.5 \%)$ & $8(11.6 \%)$ & $41(19.5 \%)$ & $56(34.4 \%)$ & $21(55.3 \%)$ & $138(21.5 \%)$ \\
$\begin{array}{c}\text { Self-rated } \\
\text { hyposmia }\end{array}$ & $8(5 \%)$ & $6(8.7 \%)$ & $19(9 \%)$ & $16(9.8 \%)$ & $11(28.9 \%)$ & $60(9.4 \%)$ \\
$\begin{array}{c}\text { Correct } \\
\text { normosmic }\end{array}$ & $143(88.8 \%)$ & $58(84.1 \%)$ & $160(76.2 \%)$ & $105(64.4 \%)$ & $14(36.8 \%)$ & $480(74.9 \%)$ \\
$\begin{array}{c}\text { Correct } \\
\text { hyposmic }\end{array}$ & $2(1.2 \%)$ & $3(4.3 \%)$ & $10(4.8 \%)$ & $14(8.6 \%)$ & $42(.1 \%)$ & $37(5.8 \%)$ \\
$\begin{array}{c}\text { Overrater } \\
\text { Underrater }^{\mathrm{c}}\end{array}$ & $10(6.2 \%)$ & $5(7.2 \%)$ & $31(14.8 \%)$ & $2(25.8 \%)$ & $13(34.2 \%)$ & $3(7.9 \%)$ \\
\hline
\end{tabular}

$S C D$ subjective cognitive decline, naMCI non-amnestic mild cognitive impairment, $a M C I$ amnestic mild cognitive impairment, $A D$ Alzheimer's disease

${ }^{\text {a }}$ Odor identification test (OIT) score $\leq 9$

${ }^{\mathrm{b}}$ Subjective olfactory capability (SOC) value $\leq 3$

${ }^{\mathrm{c}}$ Classification according to the comparison between the odor identification test (OIT) score and the subjective olfactory capability (SOC) value 
a

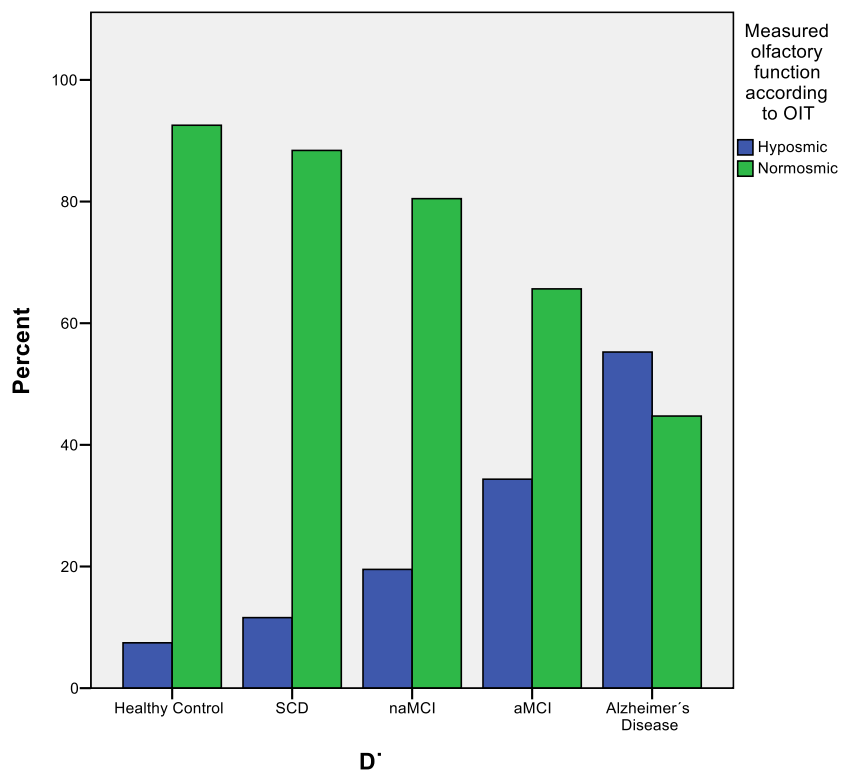

Fig. 1 a Measured olfactory function among diagnostic groups according to OIT cutoff. SCD subjective cognitive decline, naMCI non-amnestic mild cognitive impairment, aMCI amnestic mild cognitive impairment, OIT odor identification test. b Self-rated olfactory function among b

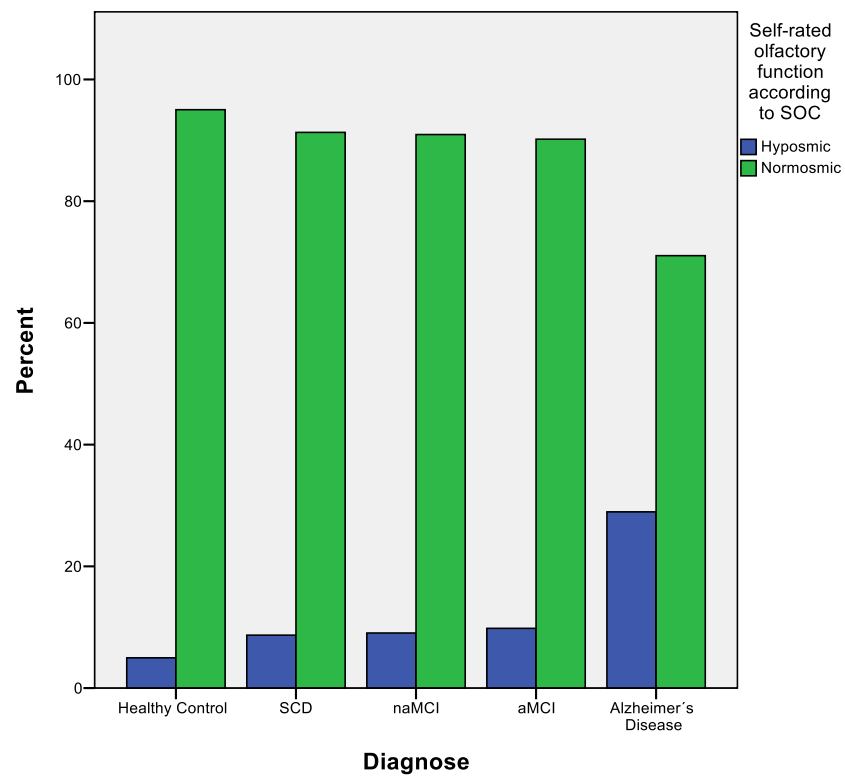

diagnostic groups according to SOC cutoff. SCD subjective cognitive decline, naMCI non-amnestic mild cognitive impairment, aMCI amnestic mild cognitive impairment, SOC subjective olfactory capability

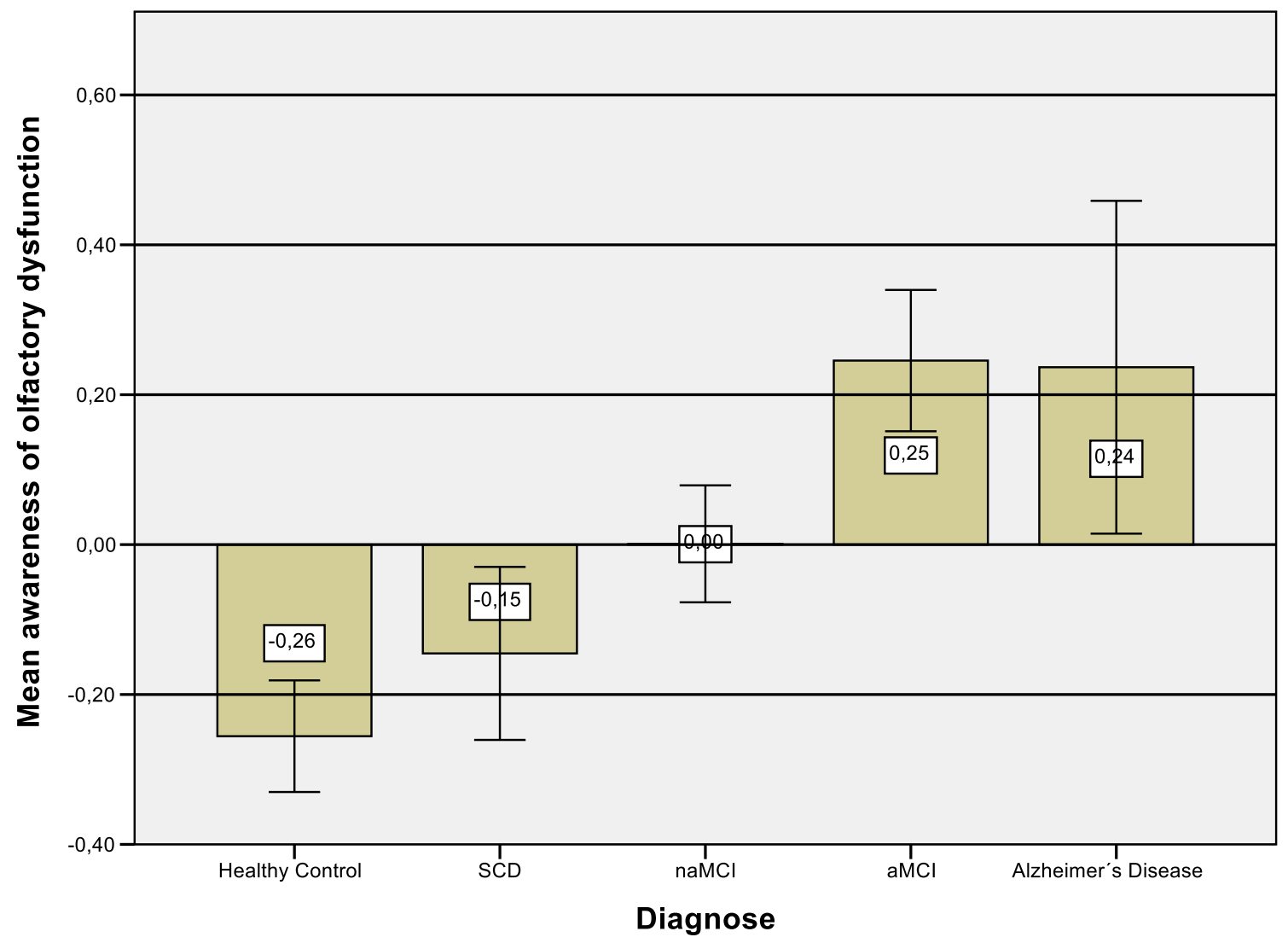

Fig. 2 Mean awareness scores of olfactory dysfunction. SCD subjective cognitive decline, naMCI non-amnestic mild cognitive impairment, aMCI amnestic mild cognitive impairment, AD Alzheimer's disease, SE standard error, OIT odor identification test, SOC subjective olfactory

capability. Awareness $=z$ score SOC $-z$ score OIT. Higher scores indicate an overestimation of olfactory function. Values close to zero indicate close similarity between the rating and the measure of olfactory function. Lower scores indicate an underestimation of olfactory function 
separately with the AD and the control group in order to compare the validity of the SOC scale between the two. The analysis with patients with $\mathrm{AD}$ resulted in an area under the curve of 0.69 ( $p=0.051,95 \% \mathrm{CI}=0.51$ to 0.86$)$, suggesting that the subjective scale did not discriminate between AD patients with measured hyposmia/anosmia and those with normosmia to a significant extent. The matching ROC curve is depicted in Fig. 3a. Figure $3 b$ shows the ROC curve that resulted from the analysis with control subjects. The respective area under the curve was $0.74(p=0.005,95 \% \mathrm{CI}=0.61-0.88)$, representing a moderate (poor to good) discriminative validity. Differences in the $\mathrm{ROC}$ areas between the $\mathrm{AD}$ and the control group were not statistically significant, since the confidence intervals overlapped.

\section{Links Between the Odor Identification Test and the SOC Domain}

To explore the relationship between the OIT and the SOC domain in the diagnostic groups, six Spearman rank correlations were executed and assessed according to the classification suggested by Cohen $(r=0.1$ being a small, $r=0.3$ being a medium, and $r=0.5$ being a large effect size) (Cohen 1988). The group of participants with SCD $\left(r_{s}=0.35, p=0.003\right)$, $\operatorname{aMCI}\left(r_{s}=0.30, p<0.001\right)$, and $\mathrm{AD}\left(r_{s}=0.40, p=0.013\right)$ showed a significant moderate correlation of the OIT score and the SOC domain, Furthermore, there was a small but also significant correlation between the two measures in the

a

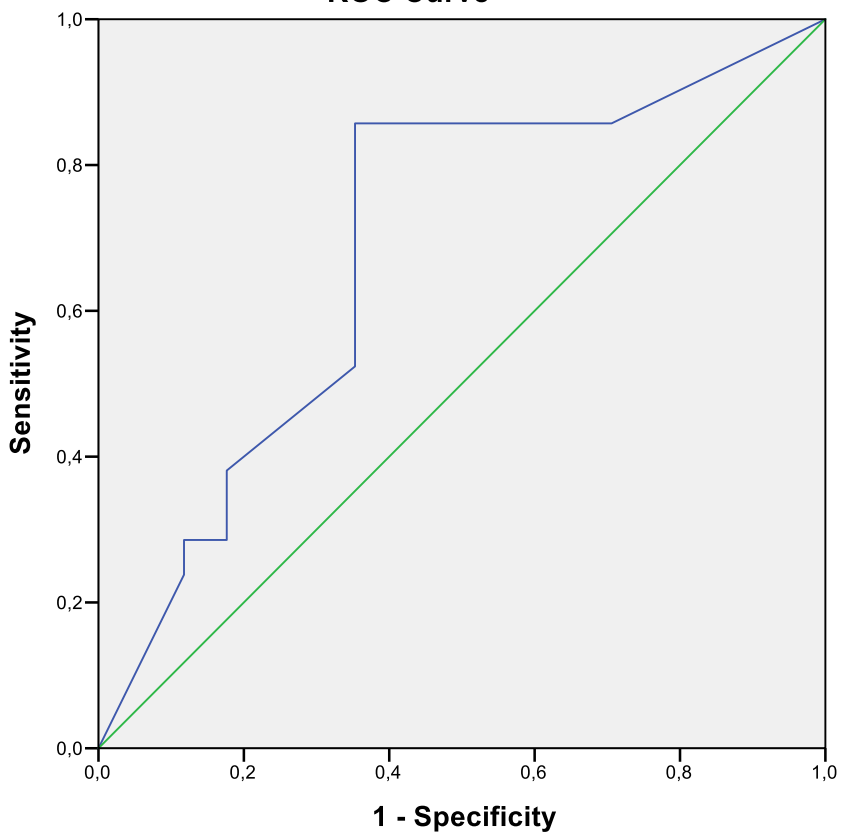

Fig. 3 a Alzheimer's disease (AD) patients' receiver operating characteristic (ROC) curves for the subjective olfactory capability (SOC) domain based on the odor identification test (OIT). AD patients with measured hyposmia/anosmia considered as cases. b Controls' receiver operating control group $\left(r_{s}=0.25, p=0.002\right)$, the naMCI group $\left(r_{s}=\right.$ $0.22, p=0.001)$, and the entire study population $\left(r_{s}=0.29\right.$, $p<0.001)$.

\section{Influential Factors on Olfactory Functioning and Its Awareness}

Concerning the links between the tested and self-rated ability to smell and aging, gender, depression as well as cognitive functioning, Table 4 displays the median, 25th and 75th percentile of age, BDI-II, VSRT delayed recall, and TMT B-A scores, and the percentage of females within the four awareness groups of the study population. In order to evaluate differences in the aforementioned metric variables between the awareness groups, a Kruskal-Wallis test was conducted, showing a significant result for all variables. To determine any significant differences between two groups, Dunn's test was applied. It revealed a significantly higher age and worse verbal memory of correct hyposmic and overrating patients compared with the other groups and significantly worse executive control functions of overraters compared with underraters and correct normosmic patients and of correct hyposmic compared with correct normosmic participants. Depressive symptoms, as measured by the BDI-II, did not differ significantly during the pairwise group comparisons. A comparison of female and male patients concerning their awareness of olfactory functioning showed a significant difference $\left(\chi^{2}(3)=15.69, p=0.001\right)$, with female patients

b

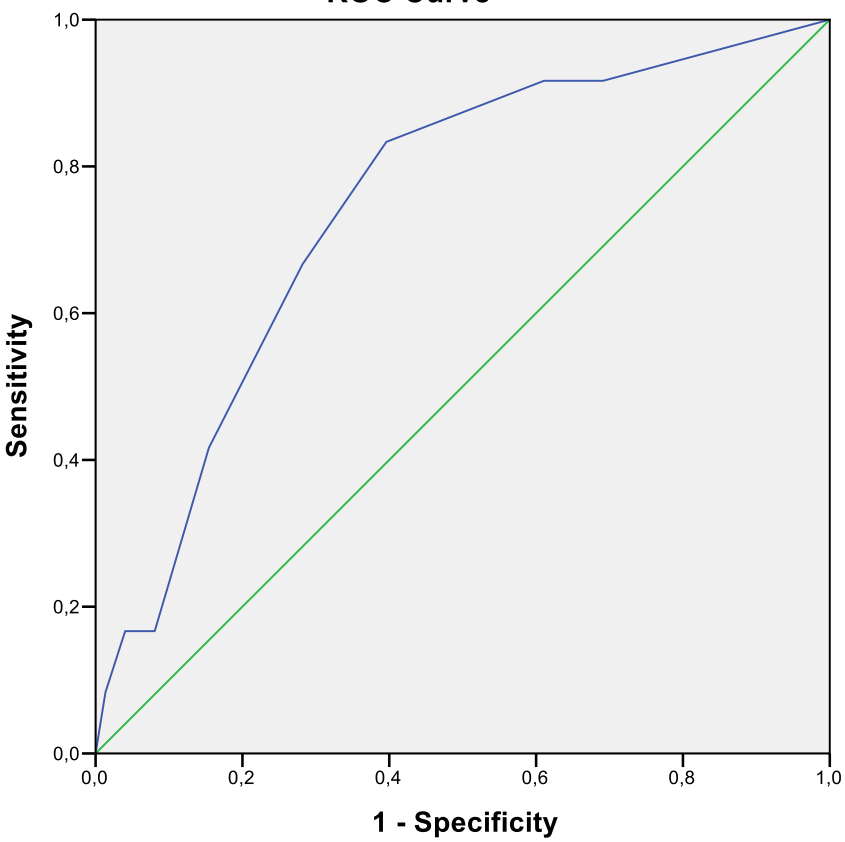

characteristic (ROC) curves for the subjective olfactory capability (SOC) domain based on the odor identification test (OIT). Controls with measured hyposmia/anosmia considered as cases 
Table 4 Comparison of age, depressive symptoms, memory, and executive control within the awareness groups of the study sample

Median (25th percentile/75th percentile)

$p$ value of Kruskal-Wallis test

Underrater $(n=23)$ Correct normosmic $(n=480)$ Correct hyposmic $(n=37)$ Overrater $(n=101)$

\begin{tabular}{llllll}
\hline Age & $61(53 / 73)^{\mathrm{c}, \mathrm{d}}$ & $67(59 / 73)^{\mathrm{c}, \mathrm{d}}$ & $73(70 / 78)^{\mathrm{a}, \mathrm{b}}$ & $75(68 / 79)^{\mathrm{a}, \mathrm{b}}$ & $<0.001$ \\
Sex & $30.4 \%$ Female $^{\mathrm{b}}$ & $62.5 \%$ Female $^{\mathrm{a}}$ & $40.5 \%$ Female & $56.4 \%$ Female & NA $^{*}$ \\
BDI-II & $10(3 / 18)$ & $7(3 / 12)$ & $9(5 / 14.5)$ & $9(5 / 14)$ & 0.021 \\
VSRT del. Recall & $11(7 / 13)^{\mathrm{c}, \mathrm{d}}$ & $11(8 / 13)^{\mathrm{c}, \mathrm{d}}$ & $7(5 / 9.5)^{\mathrm{a}, \mathrm{b}}$ & $8(4 / 10)^{\mathrm{a}, \mathrm{b}}$ & $<0.001$ \\
TMT B-A & $47(34 / 66)^{\mathrm{d}}$ & $49(33 / 75)^{\mathrm{c}, \mathrm{d}}$ & $70(42.5 / 109)^{\mathrm{b}}$ & $82(57 / 123)^{\mathrm{a}, \mathrm{b}}$ & $<0.001$ \\
\hline
\end{tabular}

Ranges in the study population: age 50-93, BDI-II 0-45, VSRT delayed recall 0-15, TMT B-A - 15-257

BDI-II Beck Depression Inventory II, VSRT del. recall Verbal Selective Reminding Test delayed recall, TMT Trail Making Test

${ }^{a}$ Significantly different from underraters according to Fisher's exact test ( $\alpha=0.05 / 6=0.0083$ due to Bonferroni correction) or Dunn's test

${ }^{\mathrm{b}}$ Significantly different from correct normosmic according to Fisher's exact test ( $\alpha=0.05 / 6=0.0083$ due to Bonferroni correction) or Dunn's test

${ }^{\mathrm{c}}$ Significantly different from correct hyposmic according to Fisher's exact test ( $\alpha=0.05 / 6=0.0083$ due to Bonferroni correction) or Dunn's test

${ }^{\mathrm{d}}$ Significantly different from overraters according to Fisher's exact test ( $\alpha=0.05 / 6=0.0083$ due to Bonferroni correction) or Dunn's test

${ }^{*} p=0.001$ for two-sided chi-squared test between all groups

accounting for almost two thirds of participants with awareness of normosmia and for less than one third of those who underrated their olfactory capability. A post hoc Fisher's exact test showed a significant difference in the gender distribution only for the aforementioned two groups of awareness. In order to check for the influence of age, depression, and cognitive functioning on tested and self-rated olfactory function regardless of awareness groups, Spearman rank correlations among the entire study population were calculated. The OIT showed a significant decline with higher age $\left(r_{s}=-0.390, p<0.001\right)$, increasing depression, as measured by the BDI-II $\left(r_{s}=-\right.$ $0.101, p=0.011$ ), and deterioration of executive control, as measured by the TMT B-A score $\left(r_{s}=-0.326, p<0.001\right)$, while a significant increase with better verbal memory, as measured by the VSRT score $\left(r_{s}=0.393, p<0.001\right)$, could be observed. The SOC correlated with these variables in a rather similar way, with the most notable differences being a non-significant association with executive control $\left(r_{s}=-\right.$ $0.025, p=0.528)$, a weaker one with age $\left(r_{s}=-0.116, p=\right.$ $0.003)$ and verbal memory $\left(r_{s}=0.135, p=0.001\right)$, and a slightly stronger one with depression $\left(r_{s}=-0.176\right.$, $p<0.001)$ compared with that of the OIT. From these significant correlations, only the ones of the OIT with age, verbal memory, and executive control were moderate in size, with the others displaying low effect sizes only. A correction for multiple testing was skipped due to the explorative nature of these Spearman rank correlations.

\section{Discussion}

The main purpose of this study was to investigate awareness of olfactory dysfunctioning in patients with SCD, naMCI, aMCI, and AD and healthy elderly people. As hypothesized and expected from prior research (Woodward et al. 2017), participants showed an olfactory identification deficit which correlated with their cognitive decline. The same correlation could be seen when looking at the prevalence of measured and self-rated hyposmia among the diagnostic groups of the study population, although the subjective score only showed a significant difference between AD patients and controls, which did not occur during previous research (Nordin et al. 1995; Bahar-Fuchs et al. 2011), and a rather subtle increase in subjectively hyposmic patients with increasing cognitive decline. This is also reflected by the distribution of the olfactory awareness groups among the study sample, which shows a growth in overraters compared with correct hyposmic patients with increasing cognitive impairment, thus being congruent with previous research (Nordin et al. 1995; Lehrner et al. 2009; Wehling et al. 2011; Bahar-Fuchs et al. 2011).

The SOC domain of the ASOF discriminated between normosmic and hyposmic/anosmic controls, as indicated by the OIT, to a moderate extent. This was not observable for subjects with $\mathrm{AD}$, although, similar to the aMCI and SCD group, they showed a significant moderate correlation of the OIT score and the SOC domain, while the entire study group, controls, and participants with naMCI displayed a small but still significant correlation. On the one hand, a fair amount of previous research showed poor prediction of olfactory function via subjective olfactory assessments (Bahar-Fuchs et al. 2011) and a lack of correlation between self-rated and measured olfaction (Djordjevic et al. 2008; Lehrner et al. 2009). On the other hand, a study by Pusswald et al., which also used the OIT and SOC for assessing olfaction, found significant results, with low and moderate correlations for controls and hyposmic patients, respectively, indicating that those differences might be due to methodical issues (Pusswald et al. 2012). This issue is further emphasized by previous research 
finding a strong correlation among patients with olfactory impairment (Welge-Luessen et al. 2005) and, if olfactory testing preceded the rating of olfactory function, also for healthy subjects (Landis et al. 2003). Although the SOC score did not show a significant ROC for detecting measured hyposmia in patients with $\mathrm{AD}$, the importance of subjective olfactory assessments in the diagnosis and prediction of AD is yet to be fully evaluated, especially when taking the relationship between the overestimation of olfactory capability and the severity of cognitive impairment among our study sample into account, which has been shown to exist in previous studies (Devanand et al. 2000).

Although we found higher age and cognitive impairment assessed via verbal memory and executive function to be more common among overraters and correct hyposmic patients, as reported by previous studies (Wehling et al. 2011, 2016; Doty and Kamath 2014), the lack of awareness of olfactory impairment per se did not correlate with these parameters, since overraters did not differ from correct hyposmic subjects, thus contradicting earlier studies (Nordin et al. 1995; Devanand et al. 2000; Wehling et al. 2011). Consequently, it may not be the metacognition of olfactory functioning but the measurably reduced sense of smell that should be focused on when looking for markers of cognitive decline. The fact that they seem to be interrelated in the current sample could partly be explained by the same underlying pathological changes within the brain. Among others, cholinergic denervation is suspected to be involved in the development of hyposmia in several neurodegenerative diseases (Doty 2017) and was found to be correlating with executive impairment (Behl et al. 2007) and overall cognitive dysfunction (Kim et al. 2013) within AD. Further investigations including data from several neurodegenerative diseases are needed to explore this potentially causative relationship (Doty 2017).

Even though gender was significantly different among awareness groups, the only significant result during post hoc testing was the lower prevalence of females among underraters (approximately one third) compared with correct normosmic participants (approximately two thirds). Prior research showed worse olfactory identification (Brämerson et al. 2004; Kern et al. 2014) and a more frequent lack of awareness of said impairment among males compared with females (Wehling et al. 2011). There is, however, no previous research regarding the influence of gender on the selfestimation of olfaction among normosmic subjects, which would be needed for a comparison with our results.

Concerning depressive symptoms, we found them to be significantly different among the awareness groups, with correct normosmic participants having the lowest and underraters having the highest median of the BDI-II score. There was, however, no significant result during the pairwise group comparisons for this variable. Prior research showed a strong association between subjective smell impairment and major depression (Hur et al. 2018). Furthermore, a review by Rochet et al. argues that measured olfactory identification is often not impaired in depression (Rochet et al. 2018). Taken together, this implies that correct normosmic patients are less likely to be depressive, especially compared with underraters, thus matching the descriptive statistics of our study sample. The lack of a significant result between correct normosmic and underrating patients might be due to the low number of participants in the latter group.

Interestingly, almost $89 \%$ of healthy elderly neither had a measured nor a self-rated impairment concerning their sense of smell. Given the findings of Murphy et al. and Wehling et al., which support the hypothesis that measured and to a smaller extent also self-rated olfactory impairment are not uncommon among otherwise healthy elderly populations, the current prevalence rate of unimpaired controls seems surprisingly high (Murphy et al. 2002; Wehling et al. 2011). Yet, the correlation between the self-rating and the measure of olfaction was small in this sample. A corresponding discrepancy was to be expected (Nordin et al. 1995; Wehling et al. 2011), although to a smaller extent in controls than in AD patients, who actually exhibited a higher correlation in this regard. This could be explained by an increased availability of information within the patient population. When only considering the awareness groups that are measurably hyposmic/ anosmic, namely the correct hyposmic and the overrating subjects of both the AD and the control group, it becomes clear that, among those having an impaired sense of smell, the share of those being fully aware of it is $38 \%$ in patients with AD but only $17 \%$ in the population of healthy elderly. Prior research by Nordin et al. found a rather identical (74\% vs $77 \%$ ) rate of unawareness of olfactory deficits among AD patients and healthy controls in their study population (Nordin et al. 1995). The higher rate of overestimating controls in our study sample could be explained by methodical differences, such as the tests used for classification of olfaction, which would also explain the different prevalences of self-rated and measured olfactory impairment among controls and $\mathrm{AD}$ patients of the study samples. This further demonstrates the need for standardized olfactory assessments in order to facilitate comparisons and improve the replicability of future studies.

There are several limitations that have to be considered when interpreting our results. Since the study design was cross-sectional, no conclusions concerning causality can be drawn. The size of the individual diagnostic groups was not equal, with a rather small number of SCD and especially AD patients reducing the feasibility of the statistical analysis. Moreover, gender and age were significantly different in the diagnostic groups, and variables such as medication intake and a history of smoking were not considered in this study, although they might have had an impact on the smelling ability. Generally, measuring olfactory functioning by means of an odor identification test requires the abilities to not only sense 
an odor but also to identify and name it which involves higher cognitive domains such as executive functions and semantic memory (Hedner et al. 2010). This means that failing to identify a certain amount of odors might still imply that the individual is able to smell the scents but has difficulties to recognize and discriminate them within the four-alternative forcedchoice format. Therefore, it is likely that cognitive capabilities had an impact on this measure and future studies should include odor threshold and discrimination testing as well. Additionally, the use of special olfactory assessments, which rely on measuring objective parameters such as sniff pressure only (Frank et al. 2006; Dulay et al. 2008), could be helpful for minimizing the effects of cognitive decline on measured olfactory capability. It can be argued that self-rated olfactory functioning was influenced by a similar methodological issue in our investigation, since it was measured by only one rating scale, with the possibility that the grading from zero to ten was too demanding for self-assessment. In the future, a more simple choice of answers such as increased, normal, or decreased sense of smell (Wehling et al. 2011) might be suitable to use, together with a scale covering the perception of specific odors like the self-reported capability of perceiving specific odors scale (SRP) of the ASOF (Pusswald et al. 2012). Another limitation is the dichotomization of measured and self-rated olfactory functioning to obtain awareness groups. Since anosognosia can be described by a continuum ranging from mild to severe deficits in self-awareness (Ries et al. 2007), the categorization into either aware or unaware represents a loss of information. Furthermore, no correction for multiple testing was applied to the correlation analysis between measured and self-rated olfaction among diagnostic groups due to the explorative nature of the analysis. And lastly, no inferential statistics could be conducted for the comparison of the prevalences of olfactory function and awareness among diagnostic groups due to the low number of participants in the individual groups.

To summarize, the present findings demonstrate that, although to some extent people with $\mathrm{AD}$ seem to be aware of their olfactory impairment in contrast to healthy elderly, the majority is not accurate enough at assessing its full scale. Thus, overestimation of the ability to smell is a common phenomenon within $\mathrm{AD}$, and self-reported functioning on its own is not a reliable source of information. Since olfactory impairment can be seen as an early marker for neurodegenerative diseases (Barresi et al. 2012), practitioners are well advised to test the ability to identify odors rather than just asking patients if they noticed any difficulties. Moreover, measurably normosmic and hyposmic participants seem to differ from each other concerning age, executive control, and verbal memory, which does not apply when comparing groups with a similar measure but different self-rating of olfactory function.
To conclude, olfactory impairment and its unawareness might serve as a diagnostic marker for early AD. Further investigations with thorough longitudinal designs and standardized assessments are needed to validate the usefulness of olfactory awareness in the detection of early $\mathrm{AD}$ and to assess the factors influencing it.

Funding Information Open access funding provided by Medical University of Vienna.

\section{Compliance with Ethical Standards}

This study was not funded.

All procedures performed in studies involving human participants were in accordance with the ethical standards of the institutional and/or national research committee and with the 1964 Helsinki declaration and its later amendments or comparable ethical standards.

Conflict of Interest The authors declare that they have no conflict of interest.

Informed Consent Informed consent was obtained from all individual participants included in the study.

Open Access This article is distributed under the terms of the Creative Commons Attribution 4.0 International License (http:// creativecommons.org/licenses/by/4.0/), which permits unrestricted use, distribution, and reproduction in any medium, provided you give appropriate credit to the original author(s) and the source, provide a link to the Creative Commons license, and indicate if changes were made.

\section{References}

Bahar-Fuchs A, Moss S, Rowe C, Savage G (2011) Awareness of olfactory deficits in healthy aging, amnestic mild cognitive impairment and Alzheimer's disease. Int Psychogeriatr 23:1097-1106. https:// doi.org/10.1017/S1041610210002371

Barresi M, Ciurleo R, Giacoppo S, Foti Cuzzola V, Celi D, Bramanti P, Marino S (2012) Evaluation of olfactory dysfunction in neurodegenerative diseases. J Neurol Sci 323:16-24. https://doi.org/10.1016/j. jns.2012.08.028

Behl P, Bocti C, Swartz RH, Gao FQ, Sahlas DJ, Lanctot KL, Streiner DL, Black SE (2007) Strategic subcortical hyperintensities in cholinergic pathways and executive function decline in treated Alzheimer patients. Arch Neurol 64:266-272. https://doi.org/10. 1001/archneur.64.2.266

Brämerson A, Johansson L, Ek L et al (2004) Prevalence of olfactory dysfunction: the skovde population-based study. Laryngoscope 114: 733-737. https://doi.org/10.1097/00005537-200404000-00026

Cohen J (1988) Statistical power analysis for the behavioral sciences, 2nd edn. Lawrence Erlbaum Associates, Hillsdale

Devanand DP, Michaels-Marston KS, Liu X, Pelton GH, Padilla M, Marder K, Bell K, Stern Y, Mayeux R (2000) Olfactory deficits in patients with mild cognitive impairment predict Alzheimer's disease at follow-up. Am J Psychiatry 157:1399-1405. https://doi.org/10. 1176/appi.ajp.157.9.1399

Devanand DP, Lee S, Manly J, Andrews H, Schupf N, Doty RL, Stern Y, Zahodne LB, Louis ED, Mayeux R (2015) Olfactory deficits predict cognitive decline and Alzheimer dementia in an urban community. 
Neurology 84:182-189. https://doi.org/10.1212/WNL. 0000000000001132

Djordjevic J, Jones-Gotman M, De Sousa K, Chertkow H (2008) Olfaction in patients with mild cognitive impairment and Alzheimer's disease. Neurobiol Aging 29:693-706. https://doi.org/ 10.1016/j.neurobiolaging.2006.11.014

Doty RL (2017) Olfactory dysfunction in neurodegenerative diseases: is there a common pathological substrate? Lancet Neurol 16:478-488

Doty RL, Kamath V (2014) The influences of age on olfaction: a review. Front Psychol 5:20

Dulay MF, Gesteland RC, Shear PK, Ritchey PN, Frank RA (2008) Assessment of the influence of cognition and cognitive processing speed on three tests of olfaction. J Clin Exp Neuropsychol 30:327337. https://doi.org/10.1080/13803390701415892

Folstein MF, Folstein SE, McHugh PR (1975) "Mini-mental state". A practical method for grading the cognitive state of patients for the clinician. J Psychiatr Res 12:189-198. https://doi.org/10.1016/ 0022-3956(75)90026-6

Frank RA, Gesteland RC, Bailie J, Rybalsky K, Seiden A, Dulay MF (2006) Characterization of the Sniff magnitude test. Arch Otolaryngol Head Neck Surg 132:532-536. https://doi.org/10. 1001/archotol.132.5.532

Hautzinger M, Keller F, Kühner C (2006) The Beck Depression Inventory II. German adaptation and manual for the BDI-II. Harcourt Test Services, Frankfurt am Main

Hedner M, Larsson M, Arnold N, Zucco GM, Hummel T (2010) Cognitive factors in odor detection, odor discrimination, and odor identification tasks. J Clin Exp Neuropsychol 32:1062-1067. https://doi.org/10.1080/13803391003683070

Hummel T, Sekinger B, Wolf SR, Pauli E, Kobal G (1997) 'Sniffin' Sticks': olfactory performance assessed by the combined testing of odour identification, odor discrimination and olfactory threshold. Chem Senses 22:39-52. https://doi.org/10.1093/chemse/22.1.39

Hummel T, Kobal G, Gudziol H, Mackay-Sim A (2007) Normative data for the "Sniffin" Sticks including tests of odor identification, odor discrimination, and olfactory thresholds: an upgrade based on a group of more than 3,000 subjects. Eur Arch Otorhinolaryngol 264:237-243. https://doi.org/10.1007/s00405-006-0173-0

Hur K, Choi JS, Zheng M (2018) Association of alterations in smell and taste with depression in older adults. Laryngoscope Investig Otolaryngol 3:94-99. https://doi.org/10.1002/lio2.142

Jessen F, Amariglio RE, van Boxtel M, Breteler M, Ceccaldi M, Chételat G, Dubois B, Dufouil C, Ellis KA, van der Flier W, Glodzik L, van Harten A, de Leon MJ, McHugh P, Mielke MM, Molinuevo JL, Mosconi L, Osorio RS, Perrotin A, Petersen RC, Rabin LA, Rami L, Reisberg B, Rentz DM, Sachdev PS, de la Sayette V, Saykin AJ, Scheltens P, Shulman MB, Slavin MJ, Sperling RA, Stewart R, Uspenskaya O, Vellas B, Visser PJ, Wagner M, Subjective Cognitive Decline Initiative (SCD-I) Working Group (2014) A conceptual framework for research on subjective cognitive decline in preclinical Alzheimer's disease. Alzheimers Dement 10:844-852. https://doi.org/10.1016/j.jalz.2014.01.001

Kern DW, Wroblewski KE, Schumm LP, Pinto JM, Chen RC, McClintock MK (2014) Olfactory function in wave 2 of the national social life, health, and aging project. J Gerontol Ser B Psychol Sci Soc Sci 69:S134-S143. https://doi.org/10.1093/geronb/gbu093

Kim HJ, Moon WJ, Han SH (2013) Differential cholinergic pathway involvement in Alzheimer's disease and subcortical ischemic vascular dementia. J Alzheimers Dis 35:129-136. https://doi.org/10.3233/ JAD-122320

Kobal G, Hummel T, Sekinger B et al (1996) "Sniffin" Sticks: screening of olfactory performance. Rhinology 34:222-226

Landis BN, Hummel T, Hugentobler M et al (2003) Ratings of overall olfactory function. Chem Senses 28:691-694. https://doi.org/10. 1093/chemse/bjg061
Lehrner JP, Brücke T, Dal-Bianco P, Gatterer G, Kryspin-Exner I (1997) Olfactory functions in Parkinson's disease and Alzheimer's disease. Chem Senses 22:105-110. https://doi.org/10.1093/chemse/22.1.105

Lehrner J, Gleiß A, Maly J et al (2006) The Verbal Selective Reminding Test (VSRT). A method for the assessment of verbal memory functions. Neuropsychiatrie 20:204-214

Lehrner J, Maly J, Gleiß A et al (2007) The Vienna Neuropsychological Test Battery (VNTB) for detecting dementia: standardization, norms, and validation. Psychol Österreich 4 5:358-365

Lehrner J, Pusswald G, Gleiss A, Auff E, Dal-Bianco P (2009) Odor identification and self-reported olfactory functioning in patients with subtypes of mild cognitive impairment. Clin Neuropsychol 23:818 830. https://doi.org/10.1080/13854040802585030

Lehrner J, Kogler S, Lamm C, Moser D, Klug S, Pusswald G, Dal-Bianco P, Pirker W, Auff E (2015) Awareness of memory deficits in subjective cognitive decline, mild cognitive impairment, Alzheimer's disease and Parkinson's disease. Int Psychogeriatr 27:357-366. https:// doi.org/10.1017/S1041610214002245

McKhann G, Drachman D, Folstein M, Katzman R, Price D, Stadlan EM (1984) Clinical diagnosis of Alzheimer's disease: report of the NINCDS-ADRDA Work Group under the auspices of Department of Health and Human Services Task Force on Alzheimer's Disease. Neurology 34:939-944

Murphy C (2002) Olfactory functional testing: sensitivity and specificity for Alzheimer's disease. Drug Dev Res 56:123-131

Murphy C, Schubert CR, Cruickshanks KJ, Klein BE, Klein R, Nondahl DM (2002) Prevalence of olfactory impairment in older adults. Jama 288:2307-2312. https://doi.org/10.1001/jama.288.18.2307

Nordin S, Monsch AU, Murphy C (1995) Unawareness of smell loss in normal aging and Alzheimer's disease: discrepancy between selfreported and diagnosed smell sensitivity. J Gerontol Ser B Psychol Sci Soc Sci 50 B:187-192. https://doi.org/10.1093/geronb/50B.4. P187

Petersen RC (2004) Mild cognitive impairment as a diagnostic entity. J Intern Med 256:183-194. https://doi.org/10.1111/j.1365-2796. 2004.01388.x

Pusswald G, Auff E, Lehrner J (2012) Development of a brief self-report inventory to measure olfactory dysfunction and quality of life in patients with problems with the sense of smell. Chemosens Percept 5:292-299. https://doi.org/10.1007/s12078-012-9127-7

Pusswald G, Moser D, Gleiß A, Janzek-Hawlat S, Auff E, Dal-Bianco P, Lehrner J (2013) Prevalence of mild cognitive impairment subtypes in patients attending a memory outpatient clinic-comparison of two modes of mild cognitive impairment classification. Results of the Vienna conversion to dementia study. Alzheimers Dement 9:366376. https://doi.org/10.1016/j.jalz.2011.12.009

Reitan RM (1979) Trail Making Test: manual for administration and scoring. Reitan Neuropsychol. Lab

Ries ML, Jabbar BM, Schmitz TW et al (2007) Anosognosia in mild cognitive impairment: relationship to activation of cortical midline structures involved in self-appraisal. J Int Neuropsychol Soc 13: 450-461. https://doi.org/10.1017/S1355617707070488

Rochet M, El-Hage W, Richa S et al (2018) Depression, olfaction, and quality of life: a mutual relationship. Brain Sci 8

Rosen HJ (2011) Anosognosia in neurodegenerative disease. Neurocase 17:231-241

$\mathrm{Sa} ß \mathrm{H}$, Wittchen H-U, Zaudig M (1996) Diagnostisches und Statistisches Manual Psychischer Störungen DSM-IV : Übersetzt nach der vierten Auflage des Diagnostic and Statistical Manual of Mental Disorders der American Psychiatric Association TT - Diagnostic and Statistical Manual of Mental Disor

Schmidt K-H, Metzler P (1992) WST-Wortschatztest. Diagnostica 40: 293-297

Wang YP, Gorenstein C (2013) Psychometric properties of the Beck Depression Inventory-II: a comprehensive review. Rev Bras 
Psiquiatr 35:416-431. https://doi.org/10.1590/1516-4446-20121048

Wehling E, Nordin S, Espeseth T, Reinvang I, Lundervold AJ (2011) Unawareness of olfactory dysfunction and its association with cognitive functioning in middle aged and old adults. Arch Clin Neuropsychol 26:260-269. https://doi.org/10.1093/arclin/acr019

Wehling EI, Wollschlaeger D, Nordin S, Lundervold AJ (2016) Longitudinal changes in odor identification performance and neuropsychological measures in aging individuals. Neuropsychology 30 : 87-97. https://doi.org/10.1037/neu0000212

Welge-Luessen A, Hummel T, Stojan T, Wolfensberger M (2005) What is the correlation between ratings and measures of olfactory function in patients with olfactory loss? Am J Rhinol 19:567-571
White TL, Kurtz DB (2003) The relationship between metacognitive awareness of olfactory ability and age in people reporting chemosensory disturbances. Am J Psychol 116:99-110. https://doi. org/10.2307/1423337

White TL, Sadikot AF, Djordjevic J (2016) Metacognitive knowledge of olfactory dysfunction in Parkinson's disease. Brain Cogn 104:1-6. https://doi.org/10.1016/j.bandc.2016.01.004

Woodward MR, Amrutkar CV, Shah HC, Benedict RHB, Rajakrishnan S, Doody RS, Yan L, Szigeti K (2017) Validation of olfactory deficit as a biomarker of Alzheimer disease. Neurol Clin Pract 7:5-14. https:// doi.org/10.1212/CPJ.0000000000000293

Publisher's Note Springer Nature remains neutral with regard to jurisdictional claims in published maps and institutional affiliations. 